\title{
COMERCIO, TIPO DE CAMBIO REAL Y CRECIMIENTO ECONÓMICO
}

\author{
TRADE, REAL EXCHANGE RATE, AND GROWTH
}

ENRIQUe R. CASARES*

\begin{abstract}
Resumen
Se desarrolla un modelo de crecimiento endógeno con tres bienes, exportable, importado y no-comerciable. Se estudia la respuesta del tipo de cambio real y de la tasa de crecimiento de la economía a una disminución de la tasa arancelaria. Se muestra que una liberalización comercial debe ir acompañada de una depreciación del tipo de cambio real. Se deduce que la tasa de crecimiento de la economía se incrementa en el largo plazo. Se argumenta que la economía mexicana no siguió este comportamiento en el periodo posterior a la liberalización comercial dando por resultado un deficiente crecimiento económico.
\end{abstract}

\begin{abstract}
We develop an endogenous growth model with three goods, exportable, importable and non-tradable. We study the response of the real exchange rate and of the economy growth rate to a decrease in the tariff rate. We show that trade liberalization must be followed by a depreciation of the real exchange rate. We deduce that the growth rate of the economy increases in the long run. We affirm that the Mexican economy did not follow this behavior in the period after the trade liberalization, so the result was a deficient economic growth.
\end{abstract}

Key words: export sector, learning by doing, real exchange rate, growth.

JEL Classification: F43, O24, O41.

* Departamento de Economía, Universidad Autónoma Metropolitana-Azcapotzalco. Av. San Pablo 180, Delegación Azcapotzalco, 02200 México, D. F., México. E-mail: ercg@correo.azc.uam.mx 


\section{INTRODUCCIÓN}

La evidencia empírica muestra que existe una relación positiva entre grado de apertura y crecimiento económico en el largo plazo (véase Dollar y Kraay 2002). Sin embargo, Baldwin (2003) señala que menores barreras comerciales sí promoverán el crecimiento siempre y cuando vayan acompañadas de un tipo de cambio estable y no distorsionado así como de otras políticas económicas. ${ }^{1}$ Es decir, un tipo de cambio distorsionado, puede conducir a una disminución de la tasa de crecimiento de la economía. Por lo tanto, para entender un proceso de liberalización comercial que pueda conducir a tasas de crecimiento mayores en el corto y largo plazo, es preciso estudiar la respuesta adecuada del tipo de cambio real a una reducción de aranceles.

En un contexto agregado, el tipo de cambio real es el nivel de precios del país extranjero (en moneda nacional) dividido entre el nivel de precios del país doméstico. En un contexto desagregado de bienes comerciables y no-comerciables, el tipo de cambio real está definido como el valor ponderado del nivel de precios relativos de los bienes no-comerciables del país extranjero dividido entre el nivel de precios relativos de los bienes no-comerciables del país doméstico. Así, considerando como dado el nivel de precios relativos del país extranjero, el tipo de cambio real está inversamente relacionado con el nivel de precios relativos de los bienes no-comerciables del país doméstico. Por lo tanto, una disminución de estos precios relativos conducirá a una depreciación del tipo de cambio real.

Así, cuando el gobierno emprende una reducción de aranceles estimula al sector exportador. Sin embargo, si esta liberalización de las importaciones va acompañada de una apreciación del tipo de cambio real, el sector exportador puede ser perjudicado. El resultado final de estas dos fuerzas opuestas sobre el sector exportador podría ser negativo para la economía. Por ejemplo, como señalan Krueger y Tornell (1999), la apreciación del tipo de cambio real del peso mexicano en el periodo postliberalización redujo la rentabilidad de exportar e incrementó la rentabilidad relativa de producir bienes no-comerciables (el índice del tipo de cambio real declinó de 139 en 1987 a 79 en 1993, $1990=$ 100). Por consiguiente, el sector no-comerciable se expandió rápidamente (construcción), mientras que el sector comerciable fue incrementando sus importaciones y produciendo grandes y graves brechas en la cuenta corriente. El resultado final fue un lento crecimiento de la economía mexicana. Asimismo, Lustig (2002) menciona, para el caso mexicano, que un tipo de cambio apreciado perjudicó las exportaciones y facilitó las importaciones. ${ }^{2}$

Así, el nivel de precios relativos de los bienes no-comerciables debería ser el correcto después de una liberalización de las importaciones. Es decir, precios

1 Asimismo, Chang et al. (2005) señalan que en economías altamente distorsionadas, una liberalización comercial sí resulta en un mayor crecimiento, si va acompañada por las reformas complementarias adecuadas.

2 Es conveniente señalar los problemas estructurales de la economía mexicana mencionados en la literatura económica, en donde se afirma que el lento crecimiento de las exportaciones mexicanas fue producto de la reestructuración de la industria mexicana (como respuesta a la liberalización comercial) y de la falta de enlaces productivos del sector exportador con el resto del sector manufacturero (véase, Dussel 2000). 
relativos que estimulen al sector exportador pero sin perjudicar al sector nocomerciable, dada la interrelación existente entre los dos sectores (el 12.4 por ciento del total de insumos del sector manufacturero mexicano proviene del sector no-comerciable, véase Martínez et al. 2004).

Casares (2004) presenta un modelo de crecimiento endógeno con dos sectores, exportador e importador, en el cual, el sector exportador es el único con aprendizaje tecnológico. Así, él muestra que una liberalización comercial produce que el factor trabajo fluya lentamente al sector exportador y que la acumulación de capital aumente en este sector. Como la tasa de crecimiento del nuevo estado estacionario es mayor, la reducción arancelaria promueve el crecimiento económico.

En este artículo se desarrolla un modelo de crecimiento endógeno de una pequeña economía abierta con dos bienes comerciables y un bien no-comerciable, con la finalidad de estudiar la respuesta del tipo de cambio real y de la tasa de crecimiento de la economía a una reducción de aranceles. Turnovsky (2000a) señala la relevancia de los modelos de crecimiento con bienes comerciables y no-comerciables para el estudio del tipo de cambio real. Por lo tanto, se extiende el modelo presentado en Casares (2004) con un bien no-comerciable. En el modelo aquí presentado existen dos bienes producidos, exportable y no-comerciable, y dos bienes de consumo, importado y no-comerciable. El precio relativo del bien no-comerciable está determinado por los cambios en la oferta y la demanda de este bien. Con este modelo se estudia una liberalización comercial.

Así, cuando el arancel es disminuido, la demanda de consumo por el bien importado aumenta y la demanda de consumo por el bien no-comerciable disminuye. Esto produce que el mercado del bien no-comerciable esté en exceso de oferta y que su precio se ajuste inmediatamente para equilibrar al mercado. En consecuencia, el precio relativo del bien no-comerciable disminuye instantáneamente, así el tipo de cambio real se deprecia inmediatamente. Con un precio relativo del bien no-comerciable menor, el salario en el sector exportador es mayor que el salario en el sector no-comerciable. Así, el trabajo fluye lentamente al sector exportador. Asimismo, el ritmo de acumulación de capital aumenta en el sector exportador. En el nuevo estado estacionario, la tasa de crecimiento del producto total aumenta, así la reducción arancelaria estimula el crecimiento económico.

Note que en este modelo la reducción de aranceles va acompañada de una depreciación inmediata del tipo de cambio real, así el sector exportador es beneficiado sin contradicciones. Mientras que en la economía mexicana la liberalización comercial fue acompañada de un aumento en el nivel de precios relativos de los bienes no-comerciables. Es decir, de una apreciación del tipo de cambio real, dando por resultado bajas tasas de crecimiento. ${ }^{3}$

El artículo está organizado de la siguiente manera. En la sección 2 se desarrolla un modelo de crecimiento endógeno con dos bienes comerciables y un bien no-comerciable. En la sección 3 se define el sistema dinámico. En la sec-

3 Mendoza (2005) evalúa si la volatilidad del tipo de cambio real en México está determinado principalmente por el movimiento de los precios relativos de los bienes no-comerciables o por el movimiento de los precios relativos de los bienes comerciables. 
ción 4 se deduce analíticamente el equilibrio de estado estacionario y se estudia la dinámica de transición. En la sección 5 se presentan las conclusiones.

\section{El Modelo}

La economía tiene tres bienes: exportable, importado y no-comerciable. Se supone que el bien exportable es producido y acumulado pero no consumido, el bien importado es consumido pero no producido ni acumulado y el bien nocomerciable es producido, consumido y acumulado. Por lo tanto, existen dos sectores productivos, exportador y no-comerciable, dos bienes de consumo, importado y no-comerciable, y dos bienes acumulables, exportable y no-comerciable (los modelos con tres bienes son simplificados frecuentemente de esta manera para estudiar políticas comerciales, véase Hinke y Nsengiyumva 1999). Se considera que el sector exportador es el único que produce progreso técnico por un aprendizaje por la práctica (learning by doing). Dado que el conocimiento es un bien público, el conocimiento generado en el sector exportador puede ser utilizado en el sector no-comerciable. Asimismo, se supone que el sector exportador es más intensivo en capital que el sector no-comerciable. La economía es pequeña, así el precio del bien exportable y del bien importado son dados por el mercado internacional. Asimismo, por simplicidad, se considera que no hay movilidad internacional de capitales, así las exportaciones son iguales a las importaciones.

Se supone que existe una migración lenta de la mano de obra del sector nocomerciable al sector exportador que depende del diferencial salarial entre los dos sectores. Se define el gasto total en consumo como una facción constante del ingreso total. Se considera que el gobierno impone un arancel sobre el bien importado y que estos ingresos arancelarios son transferidos a los individuos por medio de una transferencia de suma fija (lump-sum). Se estudia el impacto de una disminución de la tasa arancelaria sobre el tipo de cambio real y la tasa de crecimiento de la economía.

\subsection{La Producción}

El bien exportable es producido por medio de capital físico, trabajo y conocimiento tecnológico. Así, la función de producción Cobb-Douglas del sector exportador es:

$$
Y_{X}=K_{X}^{\alpha} L_{X}^{1-\alpha} T_{1}
$$

en donde $Y_{X}$ es el producto en el sector exportador, $K_{X}$ es el acervo de capital físico acumulado con el bien exportable, $L_{X}$ es el trabajo empleado en el sector, $\alpha$ y $1-\alpha$ son las participaciones de $K_{X}$ y $L_{X}$ en el producto sectorial y $T_{1}$ es el conocimiento tecnológico generado en el sector exportador. Se supone que el conocimiento tecnológico es un subproducto de la inversión realizada en el sector exportador, es decir, el conocimiento es acumulado por medio de un aprendizaje por la práctica (véase Romer 1989). Así, $T_{1}=K_{X}^{1-\alpha}$ es una externalidad que mide la contribución del conocimiento en la producción del bien expor- 
table. Se hace notar que, dado el valor del exponente en la externalidad, la función de producción del sector exportador posee rendimientos constantes en el capital ampliamente medido. Así, el modelo tiene una tasa de crecimiento de largo plazo endógena.

Se define a $\bar{P}_{X}$ como el precio internacional del bien exportable, que está determinado por el mercado mundial y que es constante. Considerando que el tipo de cambio nominal es igual a uno y que es constante, tenemos que $P_{X}=\bar{P}_{X}$, en donde $P_{X}$ es el precio doméstico del bien exportable que será utilizado como numerario $\left(P_{X}=1\right)$. Se considera que en el sector exportador existe competencia perfecta y que las empresas maximizan beneficios, tomando como dado $T_{1}$. Dado que se supone que la tasa de depreciación para $K_{X}$ es cero y que el precio del bien exportable es el numerario, la renta apropiada para $K_{X}$ es su rendimiento $r_{X}$. Así, las condiciones marginales para el sector exportador son:

$$
\begin{gathered}
w_{X}=(1-\alpha) K_{X} L_{X}^{-\alpha} \\
r_{X}=\alpha L_{X}^{1-\alpha}
\end{gathered}
$$

La ecuación (2) dice que el salario en el sector exportador, $w_{x}$, es igual al valor del producto marginal de $L_{X}$. La ecuación (3) establece que el rendimiento es igual al producto marginal de $K_{X}$.

La función de producción Cobb-Douglas para el sector no-comerciable es:

$$
Y_{N}=K_{N}^{\beta} L_{N}^{1-\beta} T_{2}
$$

En donde $Y_{N}$ es el producto en el sector, $K_{N}$ es el acervo de capital físico acumulado con el bien no-comerciable, $L_{N}$ es el trabajo empleado en el sector, $\beta$ y $1-\beta$ son las participaciones de $K_{N}$ y $L_{N}$ en la producción sectorial y $T_{2}$ es el conocimiento generado en el sector exportador pero utilizado en el sector nocomerciable. Así, $T_{2}=K_{X}^{1-\beta}$ es una externalidad que mide la contribución del conocimiento en el sector no-comerciable. De nuevo, dado el valor del exponente en la externalidad, la función de producción del sector no-comerciable tiene rendimientos constantes en el capital ampliamente medido. Así, el modelo posee solución analítica.

Se define a $p_{N}$ como el precio relativo del bien no-comerciable en términos del bien exportable. Dado que la tasa de depreciación para $K_{N}$ es cero, la renta apropiada para $K_{N}$ es $\left(r_{N}-\dot{p}_{N} / p_{N}\right)$, en donde $\dot{p}_{N} / p_{N}$ es la tasa de crecimiento del precio relativo del bien no-comerciable. Por lo tanto, las condiciones marginales para el sector no-comerciable son:

$$
\begin{gathered}
w_{N}=p_{N} K_{N}^{\beta} K_{X}^{1-\beta}(1-\beta) L_{N}^{-\beta} \\
r_{N}-\frac{\dot{p}_{N}}{p_{N}}=\beta K_{N}^{\beta-1} K_{X}^{1-\beta} L_{N}^{1-\beta}
\end{gathered}
$$

La ecuación (5) establece que el salario en el sector no-comerciable, $w_{N}$, es igual al valor del producto marginal de $L_{N}$. La ecuación (6) dice que la renta de $K_{N}$ es igual al producto marginal de $K_{N}$. 
Para resolver el modelo y dado que los acervos de capital crecerán en todo momento, es necesario redefinir las variables del modelo como variables estacionarias, es decir, variables que sean constantes en el estado estacionario. Así, se define la relación entre los acervos de capital, $z=K_{N} / K_{X}$, como la primera variable estacionaria.

Definiendo a $L$ como el trabajo total de la economía, la condición de equilibrio en el mercado laboral es $L_{X}+L_{N}=L$. Se considera que el trabajo total es constante y está normalizado a 1 . Por lo tanto, la condición de equilibrio del mercado laboral se puede expresar como $n+(1-n)=1$. En donde $n$ es la fracción del trabajo empleado en el sector exportador y $(1-n)$ es la fracción del trabajo empleado en el sector no-comerciable. Como $n$ es constante en el estado estacionario, la variable $n$ se define como la segunda variable estacionaria. Asimismo, como $p_{N}$ será constante en el estado estacionario, el precio relativo del bien no-comerciable se define como la tercera variable estacionaria.

Por lo tanto, la función de producción del sector exportador en términos de variables estacionarias es:

$$
Y_{X}=K_{X} n^{1-\alpha}
$$

y las condiciones marginales para el sector exportador en términos de variables estacionarias son:

$$
\begin{gathered}
w_{X}=(1-\alpha) K_{X} n^{-\alpha} \\
r_{X}=\alpha n^{1-\alpha}
\end{gathered}
$$

Asimismo, la función de producción del sector no-comerciable en términos de variables estacionarias es:

$$
Y_{N}=K_{X} z^{\beta}(1-n)^{1-\beta}
$$

y las condiciones marginales para el sector no-comerciable en términos de variables estacionarias son:

$$
\begin{gathered}
w_{N}=p_{N} z^{\beta} K_{X}(1-\beta)(1-n)^{-\beta} \\
r_{N}=\beta z^{\beta-1}(1-n)^{1-\beta}+\frac{\dot{p}_{N}}{p_{N}}
\end{gathered}
$$

Finalmente, se supone que $\alpha>\beta$, es decir, el sector exportador es más intensivo en capital que el sector no-comerciable.

\subsection{El Mecanismo de Migración del Trabajo}

Se supone que el trabajo fluye lentamente entre los dos sectores productivos y que este flujo intersectorial depende del diferencial salarial entre los dos sectores. Por lo tanto, la ecuación que representa el mecanismo de migración intersectorial del trabajo es: 


$$
\frac{\dot{n}}{n}=\frac{b\left(w_{X}-w_{N}\right)}{w_{N}}
$$

en donde $\dot{n} / n$ es la tasa de crecimiento de $n$ y $b$ es un parámetro positivo que mide la velocidad del proceso de migración intersectorial (véase Mas-Colell y Razin 1973). Los valores de $w_{X}$ y $w_{N}$ están dados por las ecuaciones (8) y (11). Se hace notar que como el trabajo es homogéneo, la mano de obra debería recibir el mismo salario en los dos sectores. La ecuación (13) no permite una igualación instantánea de los salarios, así el mercado laboral está segmentado (véase Agénor y Santaella 1998 y Agénor 2005).

\subsection{Individuos}

El precio internacional del bien importado, $\bar{P}_{M}$, es constante y está dado por el mercado mundial. Así, el precio doméstico del bien importado es $P_{M}=(1+\tau) \bar{P}_{M}$, en donde $\tau$ es la tasa arancelaria. Por lo tanto, el precio relativo doméstico del bien importado en términos del bien exportable es $p_{M}=(1+\tau) \bar{p}_{M}$.

Así, la restricción presupuestal de los individuos a precios domésticos es:

$$
Y_{X}+p_{N} Y_{N}+T=p_{M} C_{M}+p_{N} C_{N}+I_{X}+p_{N} I_{N}
$$

en donde $Y_{X}+p_{N} Y_{N}=Y$ es el valor de la producción total, o el ingreso total, a precios internacionales, $T$ es la transferencia de suma fija procedente del gobierno, $C_{M}$ es el consumo en el bien importado, $C_{N}$ es el consumo en el bien nocomerciable, $I_{X}$ es la inversión en $K_{X}$ y $I_{N}$ es la inversión en $K_{N}$.

Por simplicidad, se supone que no existe elección intertemporal, así el gasto total en consumo a precios internacionales, $\bar{p}_{C} C$, es una fracción constante del ingreso total a precios internacionales, es decir:

$$
\bar{p}_{C} C=\hat{c}\left(Y_{X}+p_{N} Y_{N}\right)
$$

en donde $\bar{p}_{C}$ es el índice de precios relativos al consumidor a precios internacionales, $C$ es el consumo agregado y $\hat{c}$ es la propensión a consumir, que es constante y exógeno $(0<\hat{c}<1)$.

Una vez que se tiene el gasto total en consumo, se determina la composición de la canasta de consumo. Así, se supone que las demandas de consumo para el bien importado y para el bien no-comerciable resultan de la maximización de la función de utilidad $u=A C_{M}^{\gamma} C_{N}^{1-\gamma}$ sujeta a la restricción del gasto total en consumo a precios domésticos $p_{c} C=p_{M} C_{M}+p_{N} C_{N}$, en donde $A=1 /\left[\gamma^{\gamma}(1-\gamma)^{1-\gamma}\right]$ es una constante, $\gamma$ y $(1-\gamma)$ son las participaciones de $C_{M} \mathrm{y}$ $C_{N}$ en el gasto total en consumo respectivamente y $p_{c}$ es el índice de precios relativos al consumidor a precios domésticos: 


$$
p_{C}=p_{M}^{\gamma} p_{N}^{1-\gamma}=(1+\tau)^{\gamma}\left(\bar{p}_{M}\right)^{\gamma}\left(p_{N}\right)^{1-\gamma}
$$

en donde $\left(\bar{p}_{M}\right)^{\gamma}\left(p_{N}\right)^{1-\gamma}=\bar{p}_{C}$. Así, las demandas para $C_{M}$ y $C_{N}$ son:

$$
\begin{gathered}
C_{M}=\gamma \frac{p_{C} C}{p_{M}}=\gamma \frac{\bar{p}_{C} C}{(1+\tau)^{1-\gamma} \bar{p}_{M}} \\
C_{N}=(1-\gamma) \frac{p_{C} C}{p_{N}}=(1-\gamma) \frac{(1+\tau)^{\gamma} \bar{p}_{C} C}{p_{N}}
\end{gathered}
$$

en donde $\bar{p}_{C} C$ está dado por la ecuación (15). ${ }^{4}$

\subsection{El Gobierno}

El gobierno recauda aranceles en la cantidad $\tau \bar{p}_{M}\left[C_{M}\right]$. Este ingreso arancelario es transferido a los individuos por medio de una transferencia de suma fija, $T$. Por lo tanto, la restricción presupuestal del gobierno es:

$$
\tau \bar{p}_{M}\left[C_{M}\right]=T
$$

\subsection{Mercados de Bienes y Tipo de Cambio Real}

Sustituyendo la ecuación (19) en la ecuación (14), se obtiene:

$$
Y_{X}+p_{N} Y_{N}=\bar{p}_{M} C_{M}+p_{N} C_{N}+I_{X}+p_{N} I_{N}
$$

esta es la restricción de recursos de la economía a precios internacionales. Como se considera que el precio relativo $p_{N}$ es flexible, el mercado del bien no-comerciable siempre estará en equilibrio. Por lo tanto, la condición de equilibrio para este mercado es:

$$
p_{N} Y_{N}=p_{N} C_{N}+p_{N} I_{N}
$$

Utilizando la ecuación anterior y la ecuación (20), se obtiene:

$$
Y_{X}-I_{X}=\bar{p}_{M} C_{M}
$$

esta ecuación dice que el valor de las exportaciones es igual al valor de las importaciones. Esto es resultado del supuesto de que no hay movilidad internacional de capitales.

Es importante señalar que en los modelos con tres bienes existe el tipo de cambio real interno para el bien exportable y para el bien importado (véase

4 Rodseth (2000) presenta modelos con bienes comerciables y no-comerciables, en donde utiliza funciones de demanda y de consumo semejantes a las de este artículo. 
Hinke y Nsengiyumva 1999). Así, el tipo de cambio real interno para el bien exportable, $q_{X}$, es el precio del bien exportable dividido entre el precio del bien no-comerciable, $q_{X}=1 / p_{N}$. Como en el modelo aquí desarrollado, el bien exportable y el no-comerciable son los únicos bienes producidos domésticamente, el valor de $q_{X}$ da una medida de los incentivos que guían la asignación de los recursos entre los dos sectores productivos. Del mismo modo, el tipo de cambio real interno para el bien importado es el precio del bien importado dividido entre el precio del bien no-comerciable, $q_{M}=p_{M} / p_{N}$. Dado que en el modelo aquí presentado, el bien importado y el no-comerciable son los únicos bienes consumidos, este precio relativo y el ingreso total determinan las decisiones de consumo.

Asimismo, el precio absoluto del bien no-comerciable puede ser usado como un único indicador del tipo de cambio real, véase Devarajan (1999). Por lo tanto, el tipo de cambio real está inversamente relacionado con el precio relativo del bien no-comerciable del país doméstico. Así, un aumento (decremento) del precio relativo del bien no-comerciable conducirá a una apreciación (depreciación) del tipo de cambio real.

\section{El Sistema Dinámico}

Es posible formular un sistema de ecuaciones diferenciales para las tres variables estacionarias y obtener su solución. El sistema dinámico puede ser expresado como:

$$
\begin{aligned}
& \dot{z}=f_{1}\left(z, n, p_{N}\right) \\
& \dot{n}=f_{2}\left(z, n, p_{N}\right) \\
& \dot{p}_{N}=f_{3}\left(z, n, p_{N}\right)
\end{aligned}
$$

Para obtener la primera ecuación diferencial del sistema (23), se aplica logaritmo y derivada respecto al tiempo a $z=K_{N} / K_{X}$, obteniéndose:

$$
\frac{\dot{z}}{z}=\frac{\dot{K}_{N}}{K_{N}}-\frac{\dot{K}_{X}}{K_{X}}
$$

en donde $\dot{z} / z$ es la tasa de crecimiento de $z, \dot{K}_{N} / K_{N}=g_{K N}$ es la tasa de crecimiento de $K_{N}$ y $\dot{K}_{X} / K_{X}=g_{K X}$ es la tasa de crecimiento de $K_{X}$.

A continuación, se obtiene la tasa de crecimiento de $K_{N}$. Así, dividiendo la ecuación (21) entre $K_{N}$, se sustituyen las ecuaciones (7), (10), (15) y (18) en (21) y considerando que $I_{N}=\dot{K}_{N}$, se obtiene la tasa de crecimiento de $K_{N}$ en términos de variables estacionarias:

$$
\frac{\dot{K}_{N}}{K_{N}}=\frac{(1-n)^{1-\beta}}{z^{1-\beta}}-\frac{(1-\gamma)(1+\tau)^{\gamma}}{p_{N}} \frac{\bar{p}_{C} C}{K_{N}}
$$


en donde:

$$
\frac{\bar{p}_{C} C}{K_{N}}=\hat{c}\left[\frac{n^{1-\alpha}}{z}+p_{N} \frac{(1-n)^{1-\beta}}{z^{1-\beta}}\right]
$$

De manera similar se obtiene la tasa de crecimiento de $K_{X}$. Así, dividiendo la ecuación (22) entre $K_{X}$, sustituyendo las ecuaciones (7), (10), (15) y (17) en (22) y considerando que $I_{X}=\dot{K}_{X}$, se obtiene la tasa de crecimiento de $K_{X}$ en términos de variables estacionarias:

$$
\frac{\dot{K}_{X}}{K_{X}}=n^{1-\alpha}-\frac{\gamma(1+\tau)^{\gamma} z}{(1+\tau)} \frac{\bar{p}_{C} C}{K_{N}}
$$

Por lo tanto, las ecuaciones (24), (25), (26) y (27) definen la primera ecuación diferencial del sistema (23). La segunda ecuación diferencial del sistema (23) está definida por las ecuaciones (8), (11) y (13):

$$
\frac{\dot{n}}{n}=\frac{b(1-\alpha) n^{-\alpha}}{p_{N} z^{\beta}(1-\beta)(1-n)^{-\beta}}-b
$$

Por último, la tercera ecuación diferencial del sistema (23) se obtiene de la condición dinámica de arbitraje para los dos tipos de capital $\left(r_{X}=r_{M}\right)$. Es decir, el producto marginal de $K_{X}$ debe ser igual al producto marginal de $K_{N}$ más las ganancias de capital de $K_{N}$ (esta condición es válida en todo tiempo). Utilizando las ecuaciones (9) y (12) se obtiene:

$$
\frac{\dot{p}_{N}}{p_{N}}=\alpha n^{1-\alpha}-\beta z^{\beta-1}(1-n)^{1-\beta}
$$

Como se hará evidente más adelante, las variables $z$ y $n$ son variables de movimiento lento, mientras que $p_{N}$ es una variable que brinca en el momento cero. Además, es posible mostrar que la tasa de crecimiento del producto total a precios internacionales es:

$$
\frac{\dot{Y}}{Y}=\frac{Y_{X}}{Y}\left[\frac{\dot{K}_{X}}{K_{X}}+(1-\alpha) \frac{\dot{n}}{n}\right]+\frac{p_{N} Y_{N}}{Y}\left[\beta \frac{\dot{\mathrm{z}}}{\mathrm{z}}+\frac{\dot{K}_{X}}{K_{X}}-(1-\beta) \frac{\dot{n}}{n} \frac{n}{(1-n)}+\frac{\dot{p}_{N}}{p_{N}}\right]
$$

donde $Y_{X} / Y=1 /\left[1+\left(p_{N} z^{\beta}(1-n)^{1-\beta} / n^{1-\alpha}\right)\right]$ es la participación de $Y_{X}$ en el producto total y $p_{N} Y_{N} / Y=1 /\left[\left(n^{1-\alpha} / p_{N} z^{\beta}(1-n)^{1-\beta}\right)+1\right]$ es la participación de $p_{N} Y_{N}$ en el producto total.

En la siguiente sección se analizan las propiedades del estado estacionario del modelo, la dinámica de transición y cómo la economía responde a una liberalización comercial. 


\section{La Solución en el Estado Estacionario y la Dinámica de Transición}

Primero es necesario investigar la existencia del equilibrio en el estado estacionario. Así, dado que los valores de $z, n$ y $p_{N}$ son constantes en el estado estacionario, las tasas de crecimiento de $z, n$ y $p_{N}$ son igual a cero en el estado estacionario. Asimismo, dado que las tasas de crecimiento de $K_{X}, K_{N}, Y_{X}, Y_{N}$ y $Y$ dependen solamente de $z, n, p_{N}$ y parámetros, es posible afirmar que sus tasas de crecimiento son constantes en el estado estacionario y que crecen a una misma tasa $g^{*}$ de estado estacionario.

Por lo tanto, dado que $\dot{z}=0$ en el estado estacionario, se tiene que $g_{K X}=g_{K N}$, es decir:

$$
\begin{aligned}
& \frac{(1-n)^{1-\beta}}{z^{1-\beta}}-\frac{(1-\gamma)(1+\tau)^{\gamma} \hat{c}}{p_{N}}\left[\frac{n^{1-\alpha}}{z}+\frac{p_{N}(1-n)^{1-\beta}}{z^{1-\beta}}\right]= \\
& n^{1-\alpha}-\frac{\gamma z \hat{c}}{(1+\tau)^{1-\gamma}}\left[\frac{n^{1-\alpha}}{z}+\frac{p_{N}(1-n)^{1-\beta}}{z^{1-\beta}}\right]
\end{aligned}
$$

Asimismo, dado que $\dot{n}$ es igual a cero en el estado estacionario, se tiene que $w_{X}=w_{N}$, es decir:

$$
(1-\alpha) n^{-\alpha}=p_{N} z^{\beta}(1-\beta)(1-n)^{-\beta}
$$

Finalmente, como $\dot{p}_{N} / p_{N}=0$ en el estado estacionario, se tiene que:

$$
\alpha n^{1-\alpha}=\beta z^{\beta-1}(1-n)^{1-\beta}
$$

La existencia del equilibrio en el estado estacionario se comprueba con la solución del sistema de ecuaciones formado por (31), (32) y (33). Así, utilizando la ecuación (33), se obtiene:

$$
\frac{(1-n)^{1-\beta}}{z^{1-\beta}}=\frac{\alpha n^{1-\alpha}}{\beta}
$$

Sustituyendo la ecuación (34) en la ecuación (31), se obtiene:

$$
\begin{aligned}
& \frac{\alpha}{\beta}-(1-\gamma)(1+\tau)^{\gamma} \hat{c}\left[\frac{1}{p_{N} z}+\frac{\alpha}{\beta}\right]= \\
& 1-\left[\frac{\gamma}{(1+\tau)^{1-\gamma}} \hat{c}\right]\left[1+p_{N} z \frac{\alpha}{\beta}\right]
\end{aligned}
$$


Ahora, es conveniente encontrar el valor del término $p_{N} z$ que aparece en la ecuación (35). Así, utilizando las ecuaciones (32) y (33), se obtiene:

$$
p_{N}=\frac{(1-\alpha)}{(1-\beta)}\left(\frac{\alpha}{\beta}\right)^{\beta /(1-\beta)} \frac{1}{n^{(\alpha-\beta) /(1-\beta)}}
$$

Asimismo, por medio de la ecuación (33), se obtiene:

$$
z=\left(\frac{\beta}{\alpha}\right)^{1 /(1-\beta)} \frac{(1-n)}{n^{(1-\alpha) /(1-\beta)}}
$$

Multiplicando la ecuación (36) por (37), se determina el término $p_{N} z$. Finalmente, sustituyendo el término $p_{N} z$ en la ecuación (35), se obtiene el siguiente polinomio:

$$
v^{2}+D v+E=0
$$

en donde $v=(1-n) / n$. El coeficiente $D$ del polinomio es:

$$
D=\frac{1}{B}\left[\frac{\beta \gamma(1+\tau)^{\gamma-1} \hat{c}+(\alpha-\beta)-(1-\gamma)(1+\tau)^{\gamma} \hat{c} \alpha}{\gamma(1+\tau)^{\gamma-1} \hat{c} \alpha}\right]
$$

en donde el término $B$ es una constante positiva:

$$
B=\frac{(1-\alpha)}{(1-\beta)}\left(\frac{\alpha}{\beta}\right)^{\beta /(1-\beta)}\left(\frac{\beta}{\alpha}\right)^{1 /(1-\beta)}
$$

Asimismo, el coeficiente $E$ del polinomio es:

$$
E=(1+\tau) \frac{(\gamma-1) \beta}{B^{2} \gamma \alpha}
$$

Analizando el signo de los coeficientes del polinomio, se puede afirmar que el coeficiente $D$ puede ser positivo o negativo y el coeficiente $E$ es negativo, dado que $(\gamma-1)<0$. Las raíces del polinomio son $v_{1}, v_{2}=\left[-D \pm\left(D^{2}-4 E\right)^{1 / 2}\right] / 2$. Ahora, dado que $E$ es negativo, en el polinomio siempre habrá una sola variación de signo en sus coeficientes, independientemente si $D$ es positivo o negativo. Asimismo, dado que existirán dos raíces reales, y dado que $E=v_{1} \cdot v_{2}$ es negativo, las raíces serán de signo opuesto. Por lo tanto, habrá exactamente una raíz positiva y una sola solución económicamente factible. 
Una vez obtenido el valor de $v=(1-n) / n$, se obtiene el valor de $n^{*}$ de estado estacionario por medio de $n^{*}=1 /(1+v)$. Dado el valor de $n^{*}$, por medio de las ecuaciones (36) y (37), se obtienen los valores de $p_{N}^{*}$ y $z^{*}=K_{N} / K_{X}$ de estado estacionario. Así, se ha demostrado la existencia del equilibrio en el estado estacionario.

Ahora es conveniente explicar, en el estado estacionario, el movimiento de las variables cuando el gobierno emprende una liberalización comercial, es decir, cuando la tasa arancelaria es reducida a cero. Así, en el nuevo estado estacionario, el precio relativo del bien no-comerciable es menor. Esto produce que haya menores recursos en el sector no-comerciable. Por lo tanto, la fracción de trabajo empleado en el sector exportador aumenta y el valor de $z=K_{N} / K_{X}$ disminuye. Finalmente, con mayores recursos en el sector exportador, la tasa de crecimiento del producto total aumenta en el nuevo estado estacionario. Así, en este modelo, la liberalización comercial estimula el crecimiento económico.

Se presenta el siguiente caso numérico ilustrativo. Los valores de los parámetros son: $\alpha=0.4, \beta=0.2, \gamma=0.3, \hat{c}=0.9$ y $b=0.2$. Cuando el gobierno impone una tasa arancelaria del 20 por ciento $(\tau=0.20)$, el resultado numérico es: $z^{*}=0.993 ; n^{*}=0.225, p_{N}^{*}=1.293 \mathrm{y} g^{*}=6.2$ por ciento anual. Cuando $\tau=0$, se obtiene: $z^{*}=0.856 ; n^{*}=0.259 ; p_{N}^{*}=1.249$ y $g^{*}=6.75$ por ciento anual. Como se observa, el precio relativo del bien no-comerciable disminuye, dando por resultado que el valor de $z$ disminuya y el valor de $n$ aumente. La tasa de crecimiento de la economía pasa de un 6.2 al $6.75 \%$ anual. Es importante señalar que en este artículo solamente se está evaluando el impacto de una reducción arancelaria, ceteris paribus, sobre las variables de la economía, así (1-n) disminuye. Sin embargo, en el proceso de desarrollo de las naciones la fracción del trabajo empleado en el sector servicios (mayormente no-comerciable) va aumentando. Para obtener resultados más adecuados con respecto a $(1-n)$ es necesario considerar en el modelo otras formas de aumento de la productividad y no solamente el aprendizaje por la práctica. Asimismo, es necesario considerar factores de demanda.

Ahora se explica la dinámica de transición. Primeramente, se linealizó el sistema dinámico (23) alrededor del equilibrio de estado estacionario. Se calcularon los valores característicos del sistema linealizado y se obtuvieron numéricamente dos raíces positivas y una raíz negativa. Por lo tanto, la dinámica del modelo es de punto silla. Como fue señalado anteriormente, las variables $z$ y $n$ son variables de movimiento lento, mientras que $p_{N}$ es una variable que brinca en el momento cero. Para que exista una única estable (no explosiva) solución es necesario que el número de raíces inestables sea igual al número de variables que brincan (véase Turnovsky 2000b). En el modelo existe una variable que brinca y una raíz positiva, por lo tanto existe una sola trayectoria (el sistema está indeterminado cuando el número de variables que brincan es diferente que el número de raíces inestables).

Así, cuando $\tau$ decrece, la demanda de consumo por el bien importado aumenta y la demanda de consumo por el bien no-comerciable disminuye. Esto produce que el mercado del bien no-comerciable se encuentre en exceso de oferta y que su precio disminuya instantáneamente para equilibrar al mercado. Con un precio relativo del bien no-comerciable menor, el salario en el sector exportador es mayor que el salario en el sector no-comerciable y la tasa de crecimiento de $n$ es positiva (véase ecuación 28). Por lo tanto, el trabajo fluye 
lentamente al sector exportador. Dado que el valor de $n$ va aumentando en el tiempo, el rendimiento de $K_{X}$ irá aumentando, ecuación (9), y el valor de $z$ irá disminuyendo a su nuevo valor de estado estacionario.

La reducción de aranceles estimula al sector exportador. Asimismo, con la disminución instantánea de $p_{N}$, se produce una depreciación inmediata del tipo de cambio real, que también estimula al sector exportador. Así, la liberalización comercial va acompañada de una devaluación del tipo de cambio real, mientras que en la economía mexicana se dio la liberalización comercial con una apreciación del tipo de cambio real entre los años 1987-1993.

\section{Conclusiones}

En este artículo se desarrolló un modelo de crecimiento endógeno con dos sectores productivos, en donde el sector exportador es el único que genera progreso técnico. El conocimiento generado en el sector exportador se utiliza en el sector no-comerciable. Estos dos sectores tienen la capacidad de acumular capital. El gasto total en consumo se divide en consumo en el bien importado y en el bien no-comerciable. Existe una migración lenta del trabajo entre los dos sectores que depende del diferencial salarial. Con el modelo se estudió la relación entre reducción de aranceles, tipo de cambio real y crecimiento económico.

Se demostró teóricamente que una reducción de aranceles debe ir acompañada inmediatamente de una disminución del precio relativo del bien no-comerciable, es decir, de una depreciación del tipo de cambio real. Así, se estimula la migración del trabajo del sector no-comerciable al sector exportador. Asimismo, se promueve la acumulación de capital en dicho sector. En el largo plazo, la economía en su conjunto tiende a una tasa de crecimiento mayor que la que tenía antes de la liberalización de las importaciones.

Se ha mencionado que economías que reducen sus aranceles y que mantienen un tipo de cambio real apreciado, tienen problemas de crecimiento económico. Esto se debe a que la reducción de la tasa arancelaria estimula al sector exportador, pero un tipo de cambio real apreciado perjudica a este sector. Estas fuerzas opuestas pueden producir bajas tasas de crecimiento. Por lo tanto, una liberalización comercial debe ir acompañada de las políticas macro y microeconómicas adecuadas que den como resultado sendas mayores de crecimiento en el tiempo para todos los sectores de la economía.

\section{REFERENCIAS}

Agénor, P. R. (2005). “The Analytics of Segmented Labor Markets”, Working Paper, University of Manchester.

Agénor, P. R. y J. A. Santaella (1998). "Efficiency Wages, Disinflation and Labor Mobility", Journal of Economic Dynamics and Control, Vol. 22; 267-291.

Baldwin, R. E. (2003). "Openness and Growth: What's the Empirical Relationship?", Working Paper 9578, NBER. 
Casares, E. R. (2004). "Liberalización Comercial, Ajuste Sectorial y Crecimiento en México, en Casares y Sobarzo (editores), Diez Años del TLCAN en México, Fondo de Cultura Económica.

Devarajan, S. (1999). "Estimates of Real Exchange Rate Misalignment with a Simple General-Equilibrium Model", en Hinkle y Montiel (editores), Exchange Rate Misalignment, Oxford University Press.

Chang, R., L. Kaltani y N. Loayza (2005). "Openness Can Be Good for Growth: The Role of Policy Complementarities", Working Paper 11787, NBER.

Dollar, D. y A. Kraay (2002). "Trade, Growth, and Poverty", Working Paper, World Bank.

Dussel, E. (2000). Polarizing Mexico: The Impact of Liberalization Strategy, Lynne Rienner Publishers.

Hinkle, L. E. y F. Nsengiyumva (1999). "The Three-Good Internal RER for Export, Imports, and Domestic Goods", en Hinkle y Montiel (editores), Exchange Rate Misalignment, Oxford University Press.

Krueger, A. y A. Tornell (1999). "The Role of Bank Restructuring in Recovering from Crises: Mexico 1995-1998, Working Paper 7042, NBER.

Lustig, N. (2002). México. Hacia la Reconstrucción de una Economía, 2a edición, El Colegio de México y Fondo de Cultura Económica.

Martínez, L., A. Tornell y F. Westermann (2004). "Globalización, Crecimiento y Crisis Financieras. Lecciones de México y del Mundo en Desarrollo", El Trimestre Económico, Vol. LXXI; 251-351.

Mas-Colell, A. y A. Razin (1973). "A Model of Intersectoral Migration and Growth", Oxford Economic Papers, Vol. 25; 72-79.

Mendoza, E. G. (2005). "Real Exchange Rate Volatility and the Price of Nontradable Goods in Economies Prone to Sudden Stops", Economia, Vol. 6; 103-135.

Rodseth, A. (2000). Open Economy Macroeconomics, Cambridge University Press.

Romer, P. M. (1989). "Capital Accumulation in the Theory of Long Run Growth", en Barro (editor), Modern Business Cycle Theory, Basil Blackwell.

Turnovsky, S. (2000a). "Growth in an Open Economy: Some Recent Developments", Working Paper 5, National Bank of Belgium.

Turnovsky, S. (2000b). Methods of Macroeconomic Dynamics, 2a edición, The MIT Press. 
